\title{
Research
}

\section{Are the serious problems in cancer survival partly rooted in gatekeeper principles?}

\author{
An ecologic study
}

\begin{abstract}
\section{Background}

It is puzzling to note that British and Danish citizens have a poorer cancer prognosis than citizens from other countries, and this study hypothesises that their low cancer survival could be partly rooted in the gatekeeper function undertaken by general practice in these two countries.
\end{abstract}

\section{Aim}

To test the association between principles of gatekeeper systems and cancer survival.

\section{Design and setting}

An ecologic study with data from EUROCARE-4 and primary care structure.

\section{Method}

This hypothesis was tested in an ecologic study on the association between three principles of gatekeeper systems and cancer survival in 19 European countries for which valid and full data were available.

\section{Results}

It was found that healthcare systems with a gatekeeper system do have a significantly lower 1 -year relative cancer survival than systems without such gatekeeper functions.

\section{Conclusion}

The possible mechanisms behind this finding are discussed, and while all the positive aspects of gatekeeping are recognised, it is strongly recommended that further research be conducted to confirm or reject the study hypothesis on this possible serious adverse effect of gatekeeping.

\section{Keywords}

cancer: Denmark; family practice, organisation; survival.

\section{INTRODUCTION}

The gatekeeper's main tasks are to be the doctor of first contact, to ensure referral of the patient in need of specialised care to the right place in the secondary healthcare system, and to coordinate the care in a patient-oriented context. The gatekeeper is typically a GP who performs primary care tasks such as diagnostic work-up, treatment of minor illnesses, screening, health promotion, rehabilitation, and followup.

The value of a strong primary care sector to a healthcare system is recognised worldwide. ${ }^{1,2}$ In many European countries, the gatekeeper system and the frequent practice of having patients on a GP list to ensure continuity of care ${ }^{3}$ have been seen as critical to cost-effective, ${ }^{4}$ patient-centred primary care as part of a comprehensive healthcare system, and as tools to ensure equal access for those in need of care. ${ }^{5}$ Therefore, in recent years, several European healthcare systems, for instance in France and Germany, have sought to strengthen their healthcare front line by assigning to the GP the role as first point of contact and gatekeeper.

Conversely, the principle of gatekeeping has never been popular in the US, where an intensive debate against gatekeeping is ongoing even in a time of general, growing consensus about the merits of a strong front line. ${ }^{7}$ The most important arguments against gatekeeping centre on the patient's lack of freedom and lack of choice, and on the detrimental effect on patient-doctor trust that springs from the doctor's

P Vedsted, MD, PhD, professor: F Olesen, GP Dr.Med.Sci, professor, The Research Unit for General Practice, Aarhus University, Aarhus, Denmark.

\section{Address for correspondence}

Peter Vedsted, The Research Unit for General Practice, Aarhus University, Bartholins Allé 2 8000 Aarhus C, Denmark.

E-mail: p.vedsteddalm.au.dk prerogative to decide on any referral to secondary care. ${ }^{7}$ Systems without gatekeepers implicitly have more easy patient-steered access to second opinion if the patients experience a need for this.

\section{Possible adverse effects of gatekeeper} systems

In a complex healthcare system, every organisational structure, like gatekeeping. may have unwanted effects. Freeman et al asked for much more research into both good and adverse effects of continuity of care.8,9 Likewise, remarkably little research has been devoted to the study of the quality of care for different patient groups in healthcare systems with and without gatekeeping

Waiting time to appropriate diagnostic work-up may be a serious side effect of gatekeeping. The present authors, like others, ${ }^{10}$ have been puzzled to note that Danish and British citizens have a poorer cancer prognosis than citizens from other countries, even though treatment regimes are apparently comparable and all of high quality. For the UK, it has been stated that 5000-10 000 patients with cancer die each year due to delays in diagnosis. ${ }^{11}$ In a Danish cohort of all incident, consecutive cancer patients, it was seen that most experienced a considerable diagnostic delay and that the delay was dominated by system-related delay. ${ }^{12,13}$ The Danish gatekeeper system has for decades been accepting long waiting lists for initial diagnosis-focused investigation of early symptoms in secondary care. This system-related

Submitted: 2 August 2010; Editor's response: 5 October 2010; final acceptance: 14 February 2011.

\section{(CBritish Journal of General Practice}

This is the full-length article (published online 1 Aug 2011) of an abridged version published in print. Cite this article as: $\mathbf{B r} \mathbf{J}$ Gen Pract 2011; DOI: 10.3399/bjgp11X588484. 


\section{How this fits in}

There is a marked difference in cancer survival between European countries; primary care has different roles in different health care systems. This study shows a significant association between lower cancer survival and primary care as gatekeeper

waiting time may cause the GPs to be reluctant to refer patients and thus to exercise inappropriate wait-and-see behaviour'.

There are other possible side effects of gatekeeping that are of relevance to cancer prognosis. Gatekeeper systems have been developing for years, partly because they have served policymakers' interests by providing the patients with the opportunity to continually contact the GP while awaiting diagnostic work-up in the secondary sector. Thus, the gatekeeper has been a political alibi for accepting long waits and therefore an alibi for fairness in cost containment; that is, rationing access to costly secondary care. Therefore, primary care has accepted long waits with many bottlenecks, and GPs have even been proud of being the costcontainment driver in the healthcare system. ${ }^{14}$ Further, a gatekeeper system may stimulate technological conservatism in the first part of a diagnostic process, as GPs are often not licensed to refer the patient directly to appropriate, advanced diagnostic tests, for instance imaging technologies. This is dubbed the principle of double gatekeeping'; that is, a system in which a patient needs to be referred to a specialist to become referred for well-indicated investigations.

Over time, gatekeeper systems may also make the GPs less responsive to their patients' medical needs, due both to their monopoly over referral and to the waiting lists for referred patients. The GPs know that each new referral will be added to an already long waiting list, and they do not want to be negatively judged by doctors in the secondary sector as referring unnecessarily. Furthermore, difficulties in getting services from secondary care may mean that GPs wait to refer patients until symptoms are more manifest.

A gatekeeper-based healthcare system may also be too rigid for proper diagnosis and care of certain categories of chronic patients who frequently cross the border between primary and secondary care. ${ }^{15,16}$

Finally, too little is known about patients willingness to take early contact to healthcare systems where they are on a gatekeeper's list compared to systems where they are free to choose a preferred provider on an ad hoc basis. Studies indicate that some people are reluctant to seek help because they are afraid of the result or ashamed, ${ }^{16-18}$ and the authors' ongoing studies seem to show that some patients 'do not want to unnecessarily bother their own well-liked and trusted doctor' (P Vedsted and F Olesen, unpublished data, 2011). This induces what could be labelled doctorinduced patient delay'. These observations invite the hypothesis that some of the principles of the gatekeeper systems may lead to adverse effects, with serious consequences for cancer survival.

\section{METHOD}

To test this hypothesis, an ecologic study was performed on the association between the gatekeeper system and cancer survival in 19 European countries for which valid and full data were available.

\section{Data and analyses}

Drawing on data from the EUROCARE-4 study, Moller et al calculated the 1-year relative cancer survival in 23 European countries. ${ }^{19}$ The 1 -year survival can be seen as a good indicator of the quality of early diagnosis and treatment. ${ }^{19}$ For each country and cancer type, they divided survival into quartiles and ranked each country according to its 1-year relative survival. Based on these results, the present study calculated a cancer survival score using an algorithm where the top quartiles were assigned the value 1, the bottom quartile the value 4 , and the two middle quartiles the value 2.5. The values for each of the 42 cancer types were then summed up for each country. Data were also collected on total 1-year relative survival from EUROCARE-4. All data concern cancer patients diagnosed in 1995-1999.

Data on primary care structures were collected from the work done by Boerma et al. ${ }^{15,20,21}$ They described European primary care in the first half of the 1990s, which makes these data suitable for establishing associations with outcome measures like cancer survival during the last half of the 1990s. The data were collected as part of a large questionnaire survey among GPs in all European countries. The GPs were asked to rate a number of clinical and structural variables and all answers were aggregated into a detailed description of general practice in each country. The explaining variables in this study were gatekeeper 
Table 1. Countries $(n=19)$ with a survival score or total relative 1 -year survival above median in relation to gatekeeper system, list system, and primary care being first point of contact

\begin{tabular}{|c|c|c|c|c|c|}
\hline & \multirow[b]{2}{*}{$n$} & \multicolumn{2}{|c|}{ Survival score } & \multicolumn{2}{|c|}{$\begin{array}{c}\text { Relative 1-year } \\
\text { survival }\end{array}$} \\
\hline & & Mediana & $P$ value $^{b}$ & Median & $P$ value ${ }^{b}$ \\
\hline \multicolumn{6}{|c|}{ Gatekeeper } \\
\hline No & 7 & 78.0 & 0.006 & 73.4 & 0.004 \\
\hline Yes & 12 & 113.3 & & 67.8 & \\
\hline \multicolumn{6}{|c|}{ List system } \\
\hline No & 9 & 85.5 & 0.006 & 73.4 & 0.004 \\
\hline Yes & 10 & 119.3 & & 66.3 & \\
\hline \multicolumn{6}{|c|}{ Primary care as first point of contact } \\
\hline Yes & 10 & 119.25 & 0.007 & 66.3 & 0.001 \\
\hline Some & 7 & 78.0 & & 73.4 & \\
\hline No & 2 & 81.0 & & 73.4 & \\
\hline
\end{tabular}

${ }^{a}$ High survival score indicated more cancer types with lowest quartile of 1-year survival. ${ }^{b}$ Non-parametric median/rank sum test, Fisher's exact test when cells with counts less than five.

\section{Funding body}

The Danish Cancer Society and the Novo Nordisk Foundation.

\section{Ethical approval}

Not applicable to this study.

\section{Provenance}

Freely submitted; externally peer reviewed.

\section{Competing interests}

The authors have declared no competing interests.

\section{Discuss this article}

Contribute and read comments about this article on the Discussion Forum: http://www.rcgp.org.uk/bjgp-discuss (yes/no), listed patients (yes/no), and primary care being the first point of contact $(1,2$, and 3 , with 1 as primary care as the first point of contact).

All 19 countries (Austria, Belgium, Denmark, England, Finland, France, Germany, Iceland, Ireland, Italy, Netherlands, Northern Ireland, Norway, Portugal, Scotland, Spain, Sweden, Switzerland, and Wales) for which data were available from both sources were included, and countries in transition at that time were excluded, that is, the former Eastern European countries, to ensure that stable healthcare systems were studied.

An ecologic study was performed, where the associations between the survival score for all cancers and gatekeeper, list system. and primary care as the first point of contact were explored. The associations were tested using non-parametric tests (median test with assignment of values equal to the median to the below-median group, which makes it equivalent to a Mann-Whitney test when comparing two groups). Linear regressions were also performed for each of the independent variables with total 1 year relative cancer survival as the dependent variable, in order to evaluate the amount of explained variation in cancer survival, using the adjusted $\mathrm{R}^{2}$. In the linear regression, adjustment was made for the proportion of cancers identified from death certificates only (DCO) and the proportion of microscopic verified cancer diagnosis included in the survival calculations. ${ }^{22}$ This was done to adjust for the potential confounding from an association between the organisation and function of the healthcare system and the fact that this could affect the survival rates. ${ }^{23}$

\section{RESULTS}

Table 1 shows the association between 1 year survivals for all cancers in relation to the healthcare system characteristics. Healthcare systems with primary care as gatekeepers, with list systems, and with primary care as the first point of contact consistently had more cancer types with low 1 -year relative cancer survival calculated as a total and as a compound index, compared with countries without these characteristics. The regression analysis adjusting for proportion of microscopic verified cancer diagnosis and proportion of DCO showed that the variables indicating the degree to which general practice is the first point of contact and whether the healthcare system makes use of patient lists explained most of the variation in survival score $\left(\mathrm{R}^{2}=0.64[P=0.010]\right.$ and $\mathrm{R}^{2}=$ $0.64[P=0.018]$, respectively). The $\mathrm{R}^{2}$ for gatekeeper was $0.65(P=0.003)$.

\section{DISCUSSION}

\section{Summary}

The study found a lower 1-year survival in healthcare systems with primary carebased gatekeeping. The results are highly consistent and support the hypothesis that a primary care position as gatekeeper, the use of patient lists, and primary care being the first point of contact with the healthcare system may, indeed, have adverse effects. The results even show rather large differences in cancer survival between the different systems labsolute difference: 5-7\%). In Denmark an increase in the relative 1-year survival rate from $66.3 \%$ to $73.4 \%$ would roughly correspond to 2000 person-years.

\section{Strengths and limitations}

Doing an ecologic study involves the risk of selecting exactly the data that produce the expected results and, especially, the risk of studying associations that are not genuinely causal or related in any plausible way (ecologic fallacy). In this study, data were collected from two totally independent sources and only countries for which data were incomplete were excluded. To increase homogeneity, transitional countries from Eastern Europe were excluded. Thus, the findings in this study represent a complete picture of all available data.

Another serious risk of ecologic studies is that the association may surface as a result of differences in data that are associated with other aspects of the healthcare system than the expected. If a gatekeeper system is also associated with poorer survival in patients with chronic diseases, this might 
partly explain the observed association. It is known that cancer patients with chronic diseases have poorer survival than patients without chronic diseases. ${ }^{24-26}$ However, if such an association should be present, it would simply strengthen the conclusion that there might be serious side effects to a gatekeeper system. Another important potential association is the completeness and validity of the used registers. If healthcare systems with gatekeepers register more cancer deaths and thus have a lower survival rate, this could be an important bias. ${ }^{23}$ It was found that the proportion of DCO was associated with the survival score (higher proportion of DCO, lower survivall and an association was also found between survival score and the proportion of microscopic verification of the diagnosis (higher proportion of verification, higher survival). Therefore, these two variables were included in the regression analysis to adjust for this important possible confounding.

The composite measure of cancer survival included all 42 cancers from the EUROCARE-4 study. However, the highest impact on total survival would be seen for the cancers with the highest incidence. Therefore, the analysis was also performed for the 10 most incident cancers (stomach, colon, rectum, lung, melanoma, breast, prostate, bladder, kidney, and nonHodgkin's lymphoma). The results (not shown) still showed a statistically significant association between the primary care structure and survival, which means that the association also holds for the 10 most incident cancers.

\section{Comparison with existing literature}

Other studies have shown that supplementary primary care physicians are associated with a better stage at diagnosis of cancer, and this indicates that it is not the focus on primary care as such that results in worse prognoses, but rather the side effects of the gatekeeper organisation. ${ }^{27-31}$ Thus, general practice can provide timely and comprehensive cancer diagnosis when given the proper conditions for, for example, diagnostic work-up and referral of patients.

\section{Implications for research and practice}

Principles of gatekeeper systems are associated with lower overall cancer survival, and this supports the study hypothesis that gatekeeping may have unexpected, serious side effects. These consequences cannot be ignored. The authors therefore strongly recommend immediate research to further explore and tackle this possible adverse effect while maintaining the best positive aspects of the gatekeeper system. Similar analyses should also be done on other diseases, to test the plausibility of the present findings. Work is needed to analyse whether the different structures studied here have an impact on the use of diagnostic investigations and the diagnostic delay experienced in the different countries. This could be done by, for example, surveying GPs in different settings about their stated usual practice (for example, vignettes) and about specific cancer patients' pathways, and by comparative register studies. Finally, the effect of giving GPs optimal access to quick specialised diagnostic help could be studied in randomised trials.

Detailed data are needed to show why the observed association may exist. However, the authors suspect that unacceptable delays in the diagnostic work-up phase, which have been an accepted tradition in most gatekeeper systems, are a major factor contributing to the low survival.

This study also draws attention to the need for more focused and comparative research on the pretreatment phase of cancer in different healthcare systems. ${ }^{11}$

In the light of the present study, it seems appropriate to rethink the role of the gatekeeper. The gatekeepers should not be viewed as/thought of as 'keepers' simply rationing care, but rather as 'advisers' who counsel the patients on what to do in a responsive healthcare system and who ensure acceptable waits for needed diagnostic work-up in secondary care. 


\section{REFERENCES}

1. Starfield $B$, Shi L, Macinko J. Contribution of primary care to health systems and health. Milbank Q 2005; 83(3): 457-502.

2. Walley J, Lawn JE, Tinker A, et al. Primary health care: making Alma-Ata a reality. Lancet 2008; 372(9642): 1001-1007.

3. Haggerty JL, Reid RJ, Freeman GK, et al. Continuity of care: multidisciplinary review. BMJ 2003; 327(7425): 1219-1221.

4. Starfield B. Is primary care essential? Lancet 1994; 344(8930): 1129-1133.

5. Allen J, Gay B, Crebolder H, et al. The European definitions of the key features of the discipline of general practice: the role of the GP and core competencies. Br J Gen Pract 2002; 52(479): 526-527.

6. Nolte E, McKee M. Measuring the health of nations: analysis of mortality amenable to health care. BMJ 2003; 327(7424): 1129.

7. Halm EA, Causino N, Blumenthal D. Is gatekeeping better than traditional care? A survey of physicians' attitudes. JAMA 1997; 278(20): 1677-1681.

8. Freeman GK, Olesen F, Hjortdahl P. Continuity of care: an essential element of modern general practice? Fam Pract 2003; 20(6): 623-627.

9. Freeman $G$, Hjortdahl P. What future for continuity of care in general practice? BMJ 1997; 314(7098): 1870-1873.

10. Hamilton W. Cancer diagnosis in primary care. Br J Gen Pract 2010; 60(571): $121-128$.

11. Richards MA. The size of the prize for earlier diagnosis of cancer in England. Br J Cancer 2009; 101(suppl 2): S125-S129.

12. Hansen RP. Delay in the diagnosis of cancer [PhD thesis]. Aarhus: Faculty of Health Sciences, University of Aarhus; 2008.

13. Olesen F, Hansen RP, Vedsted P. Delay in diagnosis: the experience in Denmark. Br J Cancer 2009; 101(suppl 2): S5-S8.

14. Goodwin N. Diagnostic delays and referral management schemes: how 'integrated' primary care might damage your health. Int J Integr Care 2008; 8: e78.

15. Boerma WG. Profiles of general practice in Europe. An international study of variation in the tasks of general practitioners [PhD thesis]. Utrecht: NIVEL; 2003.

16. Broom DH. Familiarity breeds neglect? Unanticipated benefits of discontinuous primary care. Fam Pract 2003; 20(5): 503-507.

17. Burgess CC, Potts HW, Hamed H, et al. Why do older women delay presentation with breast cancer symptoms? Psychooncology 2006; 15(11): 962-968.

18. Robb K, Stubbings S, Ramirez A, et al. Public awareness of cancer in Britain a population-based survey of adults. Br J Cancer 2009; 101(suppl 2): S18-23.

19. Møller H, Linklater KM, Robinson D. A visual summary of the EUROCARE-4 results: a UK perspective. Br J Cancer 2009; 101(suppl 2): S110-S114.

20. Boerma WG, van der ZJ, Fleming DM. Service profiles of general practitioners in Europe. European GP Task Profile Study. Br J Gen Pract 1997; 47(421): 481-486.

21. Saltman RB, Rico A, Boerma W (eds). Primary care in the driver's seat? Maidenhead: Open University Press; 2006.

22. Angelis R, Francisci $S$, Baili $P$, et al. The EUROCARE-4 database on cancer survival in Europe: Data standardisation, quality control and methods of statistical analysis. Eur J Cancer 2009; 45(6): 901-1094.

23. Robinson D, Sankila R, Hakulinen T, Moller H. Interpreting international comparisons of cancer survival: the effects of incomplete registration and the presence of death certificate only cases on survival estimates. Eur J Cancer 2007; 43(5): 909-913.

24. Cronin-Fenton DP, Norgaard M, Jacobsen J, et al. Comorbidity and survival of Danish breast cancer patients from 1995 to 2005. Br J Cancer 2007; 96(9): 1462-1468.

25. Iversen LH, Norgaard M, Jacobsen J, et al. The impact of comorbidity on survival of Danish colorectal cancer patients from 1995 to 2006 - a population-based cohort study. Dis Colon Rectum 2009; 52(1): 71-78.

26. Tetsche MS, Norgaard M, Jacobsen J, et al. Comorbidity and ovarian cancer survival in Denmark, 1995-2005: a population-based cohort study. Int J Gynecol Cancer 2008; 18(3): 421-427.

27. Campbell RJ, Ramirez AM, Perez K, Roetzheim RG. Cervical cancer rates and the supply of primary care physicians in Florida. Fam Med 2003; 35(1): 60-64.

28. Ferrante JM, Gonzalez EC, Pal N, Roetzheim RG. Effects of physician supply on early detection of breast cancer. J Am Board Fam Pract 2000; 13(6): 408-414.

29. Roetzheim RG, Pal N, Van Durme DJ, et al. Increasing supplies of dermatologists and family physicians are associated with earlier stage of melanoma detection. J Am Acad Dermatol 2000; 43(2 Pt 1): 211-218.

30. Roetzheim RG, Pal N, Gonzalez EC, et al. The effects of physician supply on the early detection of colorectal cancer. J Fam Pract 1999; 48(11): 850-858.

31. Gorey KM, Luginaah IN, Fung KY, et al. Physician supply and breast cancer survival. J Am Board Fam Med 2010; 23(1): 104-108. 